\title{
Chemotaxis of artificial microswimmers in active density waves
}

\author{
Alexander Geiseler, ${ }^{1, *}$ Peter Hänggi,,${ }^{1,2,3}$ Fabio Marchesoni, ${ }^{4,5}$ Colm Mulhern, ${ }^{1}$ and Sergey Savel'ev ${ }^{6}$ \\ ${ }^{1}$ Institut für Physik, University of Augsburg, D-86159, Germany \\ ${ }^{2}$ Nanosystems Initiative Munich, Schellingstraße 4, D-80799 München, Germany \\ ${ }^{3}$ Department of Physics, National University of Singapore, 117551 Singapore, Republic of Singapore \\ ${ }^{4}$ Center for Phononics and Thermal Energy Science, School of Physics Science and Engineering, \\ Tongji University, Shanghai 200092, People's Republic of China \\ ${ }^{5}$ Dipartimento di Fisica, Università di Camerino, I-62032 Camerino, Italy \\ ${ }^{6}$ Department of Physics, Loughborough University, Loughborough, LE11 3TU, United Kingdom
}

(Received 11 January 2016; published 15 July 2016)

\begin{abstract}
Living microorganisms are capable of a tactic response to external stimuli by swimming toward or away from the stimulus source; they do so by adapting their tactic signal transduction pathways to the environment. Their self-motility thus allows them to swim against a traveling tactic wave, whereas a simple fore-rear asymmetry argument would suggest the opposite. Their biomimetic counterpart, the artificial microswimmers, also propel themselves by harvesting kinetic energy from an active medium, but, in contrast, lack the adaptive capacity. Here we investigate the transport of artificial swimmers subject to traveling active waves and show, by means of analytical and numerical methods, that self-propelled particles can actually diffuse in either direction with respect to the wave, depending on its speed and waveform. Moreover, chiral swimmers, which move along spiraling trajectories, may diffuse preferably in a direction perpendicular to the active wave. Such a variety of tactic responses is explained by the modulation of the swimmer's diffusion inside traveling active pulses.
\end{abstract}

DOI: 10.1103/PhysRevE.94.012613

\section{INTRODUCTION}

Taxis is the biased movement of bacteria, somatic cells, or multicellular organisms in response to external stimuli such as light, electric currents, gravity, and chemicals. Taxes are classified based on the type of activating stimulus and on whether the organism's resultant drift is oriented toward (positive) or away from (negative) the stimulus source [1]. Tactic cell migration plays a crucial role in biological pattern formation and the organization of complex biological organisms. For instance, bacteria find food (e.g., glucose) or flee from poison (e.g., phenol) by swimming, respectively, up (positive chemotaxis) or down (negative chemotaxis) the concentration gradient of the sensed chemical $[2,3]$.

A biomimetic counterpart of cellular motility is the ability of specially designed synthetic microparticles to propel themselves by harvesting kinetic energy from an active environment $[4,5]$. Self-propulsion is fueled by stationary nonequilibrium processes, like directional "power-strokes" from catalytic chemical reactions or self-phoresis by shortscale (electric, thermal, or chemical) gradients, produced by the particle itself, in virtue of some built-in functional asymmetry [4-6]. Similarly to bacteria, artificial microswimmers are also known to diffuse up or down long-scale monotonic gradients of the active medium [7-10]. However, bacteria regulate their response to an external stimulus by adapting their (complex) tactic signal transduction pathways [11], which thus operate like sensor-actuator loops. In contrast, due to their often submicron size and lack of an internal structure, synthetic swimmers are unable to process the tactic signal $[12,13]$, i.e., their response being strictly local.

\footnotetext{
*alexander.geiseler@physik.uni-augsburg.de
}

In most circumstances the tactic stimulus is modulated in space and time in the form of single or entrained active pulses that sweep through the suspended swimmer. Certain microorganisms are able to locate the source of the pulses and move towards it, no matter what the sign of their response to a monotonic active gradient. A study case is the chemotactic aggregation of the amoebae of the cellular slime mold (Dictyostelium discoideum) [14]. This process is directed by periodic sequences (or waves) of symmetric concentration pulses of a chemoattractant, irradiating from the aggregation center outwards. As amoebae exhibit positive chemotaxis, one would expect a cell movement toward the center in the wave-front and away from it in the wave-back. As a consequence, amoebae would spend more time in the back wave than in the front wave, thus undergoing a net drift in the direction of the active wave propagation. Most remarkably, the conclusion of this argument (first proposed by Stokes in a more general fluidodynamics context $[15,16]$ ) does not change by reversing the sign of amoeba chemotaxis: according to Stokes's wave fore-rear asymmetry argument and contrary to experimental observations, swimmers with definite chemotaxis (positive or negative, alike) would always surf active density waves (ADW), thus moving away from the the wave source [17]. A proposed resolution of this apparent paradox (termed "chemotactic wave paradox" in Ref. [18]) for cellular microorganisms requires the standard model for cell chemotaxis to be modified so as to account for a finite adaptation time of the chemotactic pathways to temporally varying stimuli [18].

This approach cannot be extended to the case of artificial swimmers diffusing across a traveling ADW because these respond to the instantaneous activation properties of the surrounding medium with no temporal memory [19]. However, controlling the transport of synthetic submicron particles, selfpropelling in a spatiotemporally modulated active medium, is 
key to the success of nanorobotics in technological applications, like environmental monitoring, intelligent drug delivery, or even more challenging biomedical tasks [6]. With this goal in mind, we numerically investigated the diffusive dynamics of artificial microswimmers at low Reynolds numbers, subjected to traveling symmetric active pulses of different waveforms. We observed that swimmers with positive taxis in a monotonic active gradient, actually drift toward or away from the pulse source, depending on the pulse sequence. Moreover, chiral swimmers, which due to some configurational asymmetry tend to move in circles [20], may also drift orthogonally to the incoming active wave. The variability of swimmers' tactic response is proven to result more from the spatiotemporal modulation of their active motion within a traveling pulse than the pulse fore-rear gradient asymmetry.

The paper is organized as follows. In Sec. II we introduce the model of the artificial microswimmer that is to be considered. Already in this section, without resorting to the finer technical details (given later), a qualitative presentation of the key results will be given. Here the tactic responses of the swimmer to single wave pulses and also to active density waves are discussed. A more quantitative discussion of these results is given in Sec. III, where, in addition, related analytical results will be presented. Finally, in Sec. IV, the details of the methods used to obtain the results will be explained, including the numerical schemes applied to integrate the Langevin and corresponding Fokker-Planck equations.

\section{RESULTS}

\section{A. Model}

Regardless of the details of the self-propulsion mechanism, at low Reynolds numbers the diffusion of an artificial microswimmer on a surface is conveniently modeled by a set of simple Langevin equations (LE):

$$
\begin{aligned}
\dot{x} & =v(x, t) \cos \phi+\sqrt{D_{0}} \xi_{x}(t), \\
\dot{y} & =v(x, t) \sin \phi+\sqrt{D_{0}} \xi_{y}(t), \\
\dot{\phi} & =\Omega+\sqrt{D_{\phi}} \xi_{\phi}(t) .
\end{aligned}
$$

Three sources of fluctuations are explicitly incorporated in the model: two translational of intensity $D_{0}$, and one orientational of intensity $D_{\phi}$. All noises are Gaussian and stationary, with zero-mean and autocorrelation functions $\left\langle\xi_{i}(t) \xi_{j}(0)\right\rangle=$ $2 \delta_{i j} \delta(t)$, with $i, j=x, y, \phi$. As such noises result from a combination of thermal fluctuations in the suspension fluid and randomness of the propulsion mechanism, we treat the intensities $D_{0}$ and $D_{\phi}$ as independent parameters. Finally, depending on their geometry, 2D swimmers often experience an additional torque, $\Omega$, which makes them rotate counterclockwise or clockwise. Positive and negative chiral effects impact the transport properties of both biological and synthetic active swimmers [20-23].

When the swimmer floats in a homogeneous active suspension, its propulsion speed is a constant, $v(x)=v_{0}$. As detailed in Sec. IV, it then undergoes an active Brownian motion with finite persistence time, $\tau_{\phi}=1 / D_{\phi}$, length, $l_{\phi}=v_{0} \tau_{\phi}$, and bulk diffusion constant $\lim _{t \rightarrow \infty}\left\langle[x(t)-x(0)]^{2}\right\rangle=D_{0}+D_{s}$, with $D_{s}=v_{0}^{2} / 2 D_{\phi}$.
To model the effects of an active pulse sweeping through the suspension fluid, we assume that the propulsion speed, $v(x, t)$, is a local function of the physiochemical properties of the medium at the swimmer's position. For pulses propagating from left to right along the $x$ axis, this amounts to inserting in Eqs. (1) an appropriate function $v(x, t)=v(x-u t)$, where $u$ is the pulse's speed and the waveform $v(x)$ is chosen so as to describe single or entrained traveling pulses [4] with amplitude $v_{0}$. Like in Ref. [18], here we restrict our analysis to spatially symmetric waveforms, $v(x)=v(-x)$, in order to avoid additional ratchet effects $[12,17]$. The exact mechanism underlying the tactic stimulus modeled by the spatiotemporal function $v(x, t)$ does not, in principle, need to be specified; it can, for instance, be of chemo-, diffusio-, electro-, or thermophoretic nature.

A proof of concept of the tactic effects predicted in this work is sketched in Fig. 1(a). In this ideal experiment a thermophoretic swimmer, floating on a planar substrate, is irradiated by a defocused laser beam [8]. A traveling train of laser intensity pulses is created by inserting a slit screen between the light source and the substrate and sliding it at a constant speed. Since the self-propulsive speed of a thermophoretic swimmer is approximately proportional to the laser intensity [24], one can thus tailor at will the spatiotemporal modulation of the velocity field, $v(x, t)$.

For the sake of concreteness, in the following we adopt the term positive or negative chemotaxis to mean the drift of an active swimmer parallel or antiparallel to the direction of a generic incoming active pulse or wave, regardless of its nature. Moreover, we remind the reader that, like in the experimental setup of Fig. 1(a), the propulsion speed of most artificial chemotactic swimmers grows linearly with the concentration of the chemoactivants in the active suspension, whereas, as assumed in Eqs. (1), their angular diffusion stays almost the same [6]. Such swimmers, when placed in a static velocity field, that is for $v(x, t)=v(x)$, are known to diffuse up the velocity gradient $[7,26]$. Other authors [8,27] have detected the dependence of the rotational diffusion on the active density wave to be generally weaker than of the propulsion speed. Extending our analysis to an $x$-dependent $D_{\phi}$ would not alter the general conclusions of the present work, except for some more laborious technical details [28].

Finally, we stress that in the ideal experiment of Fig. 1(a) hydrodynamic effects can be largely suppressed, at least in the absence of activant gradients. Indeed [3], (i) swimmers freely diffuse in the bulk away from the container's walls; (ii) their density can be lowered so as to avoid clustering [29]; and (iii) they can be fabricated rounded in shape and so small in size (i.e., pointlike) to minimize hydrodynamic backflow effects. On the contrary, the activant gradients considered in the present work surely cause additional hydrodynamic effects in the form of a polarizing torque that tends to align the particle's velocity parallel or antiparallel to the gradient, depending on the swimmer's surface properties [30]. Our simulations show that chemotaxis is enhanced for swimmers aligned against the activant gradient and suppressed in the opposite case, as also reported in Ref. [8]. However, we numerically checked that the chemotactic effect persists even in the latter case, solely its magnitude slightly diminishes. To keep our discussion as simple as possible, polarization effects will be 

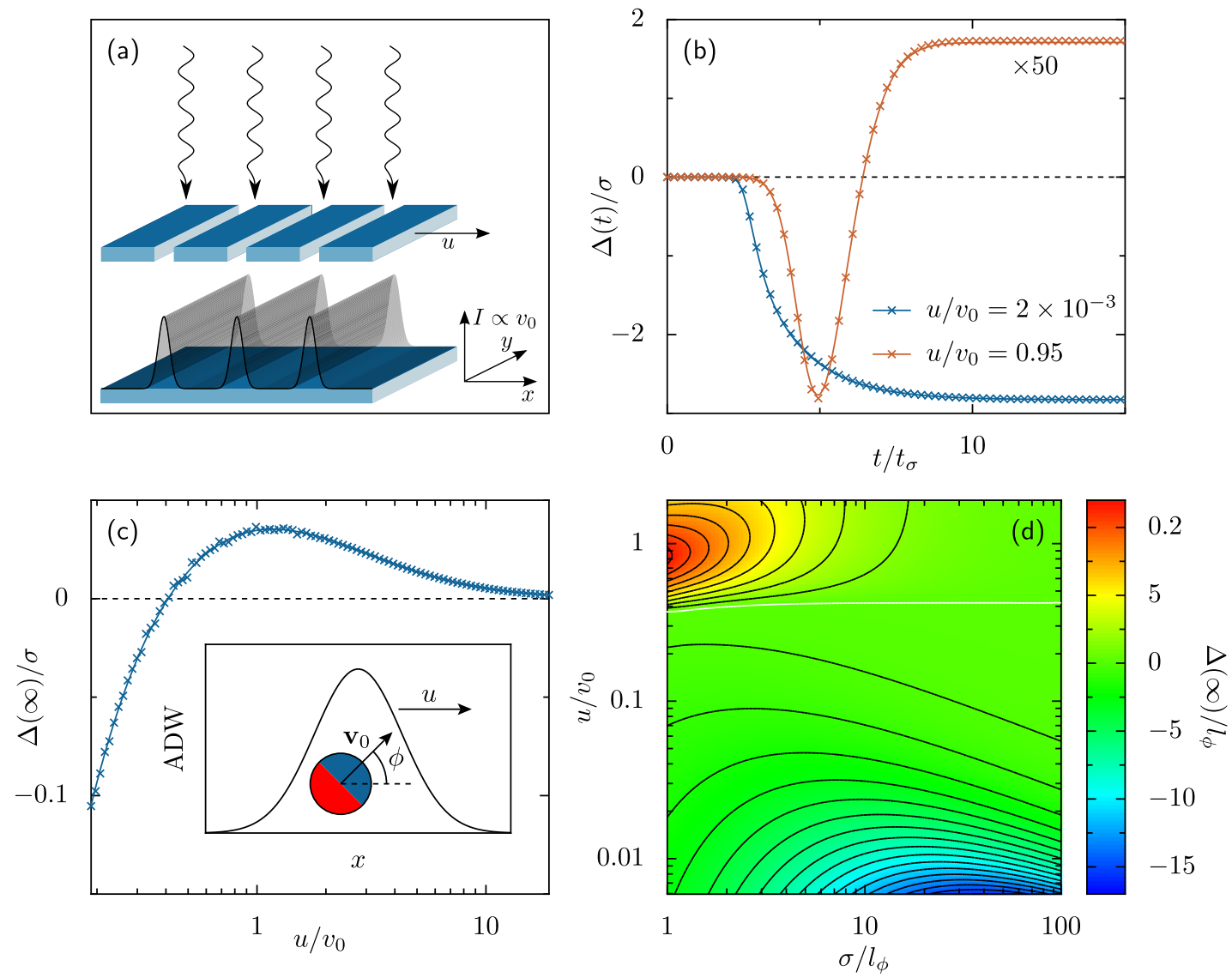

FIG. 1. (a) Ideal experimental setup of a thermophoretic swimmer diffusing on a planar substrate irradiated by a laser beam [8,25]. By pulling at constant speed a slit-screen sliding between the laser and the substrate, it is possible to modulate the laser intensity, $I$, hitting the particle, thus realizing an effective ADW. (b, c) Chemotactic shift induced by a Gaussian active pulse, $v(x)=v_{0} \exp \left(-x^{2} / 2 \sigma^{2}\right)$, traveling to the right with constant speed $u$ (see inset of (c), where a simple sketch of the model is depicted). The swimmer's self-propulsion parameters, $v_{0}=53 \mu \mathrm{m} / \mathrm{s}$ and $D_{\phi}=165 \mathrm{~s}^{-1}$, were chosen to mimic the experimental setup of Ref. [25], and the pulse width, $\sigma=1 \mu \mathrm{m}$, was set three times the swimmer's propulsion length $l_{\phi}=v_{0} / D_{\phi}$. Translational noise $D_{0}$ and chiral torque $\Omega$ were set to zero to focus on the basic mechanism responsible for the emergence of the spatial shift, $\Delta(t)=\langle x(t)-x(0)\rangle$. In panel (b) the particle displacement, $\Delta(t)$, is plotted vs. $t$ in units of the pulse crossing time $t_{\sigma}=\sigma / u$ for a slow and fast pulse. For the sake of comparison, we remind that the time the particle takes to diffuse a length $\sigma$ in the bulk is $\tau_{\sigma}=\sigma^{2} / 2 D_{s}$. In panel (c) the final displacement $\Delta(\infty)$ is plotted as a function of the pulse speed. The stochastic integration of the model Eqs. (1) (crosses) are compared with the numerical solution the corresponding Fokker-Planck Eq. (7) (solid curves). The contour plot in panel (d) illustrates the dependence of $\Delta(\infty)$ on both pulse parameters $\sigma$ and $u$. The white curve separates the regions with positive and negative $\Delta(\infty)$.

neglected here and fully investigated in a follow-up technical report.

We now qualitatively discuss the numerical results of Figs. 1-4, which were obtained by numerically integrating the LEs Eq. (1) and the corresponding Fokker-Planck equation (FPE) (see Sec. IV). A more technical analysis of these results will be presented in Sec. III.

\section{B. Chemotaxis by single active pulses}

In Figs. 1(b)-1(d) and Supplemental Material movies 1 and 2 [31] we illustrate the effects of an active Gaussian pulse of amplitude $v_{0}$ traveling from left to right across a swimmer at rest in the absence of translational fluctuations, $D_{0}=0$. The swimmer undergoes a net longitudinal shift, $\Delta(\infty)=\lim _{t \rightarrow \infty}\langle x(t)-x(0)\rangle$ : most notably, $\Delta(\infty)$ is markedly negative for slow pulses, $u \ll v_{0}$, and positive for fast pulses, $u \geqslant v_{0}$; see Figs. 1(c) and 1(d). We explain the existence of opposite chemotactic regimes by noticing that a swimmer with $D_{0}=0$ diffuses only across the width of the incoming active pulse. Suspended inside a slow traveling pulse with $u \ll v_{0}$, the particle quickly diffuses either against the front or the rear of the pulse. Upon reaching either pulse's edge, its diffusivity gets suppressed, that is its self-propulsive velocity, $v(x)$, grows smaller than the pulse speed, $u$. The ensuing behavior at the two sides of the pulse is different. After hitting the right-hand side, the particle is caught up again by the advancing pulse and resumes diffusing, whereas upon hitting the left-hand side, it is left behind and comes to rest. The two pulse's edges behave, respectively, like traveling reflecting and adsorbing walls. As a result we expect, on average, a net shift of the particle to the left. In the opposite regime of a fast traveling pulse, $u \gg v_{0}$, the particle comes almost immediately to rest when hitting the left pulse wall, while it can travel a much 

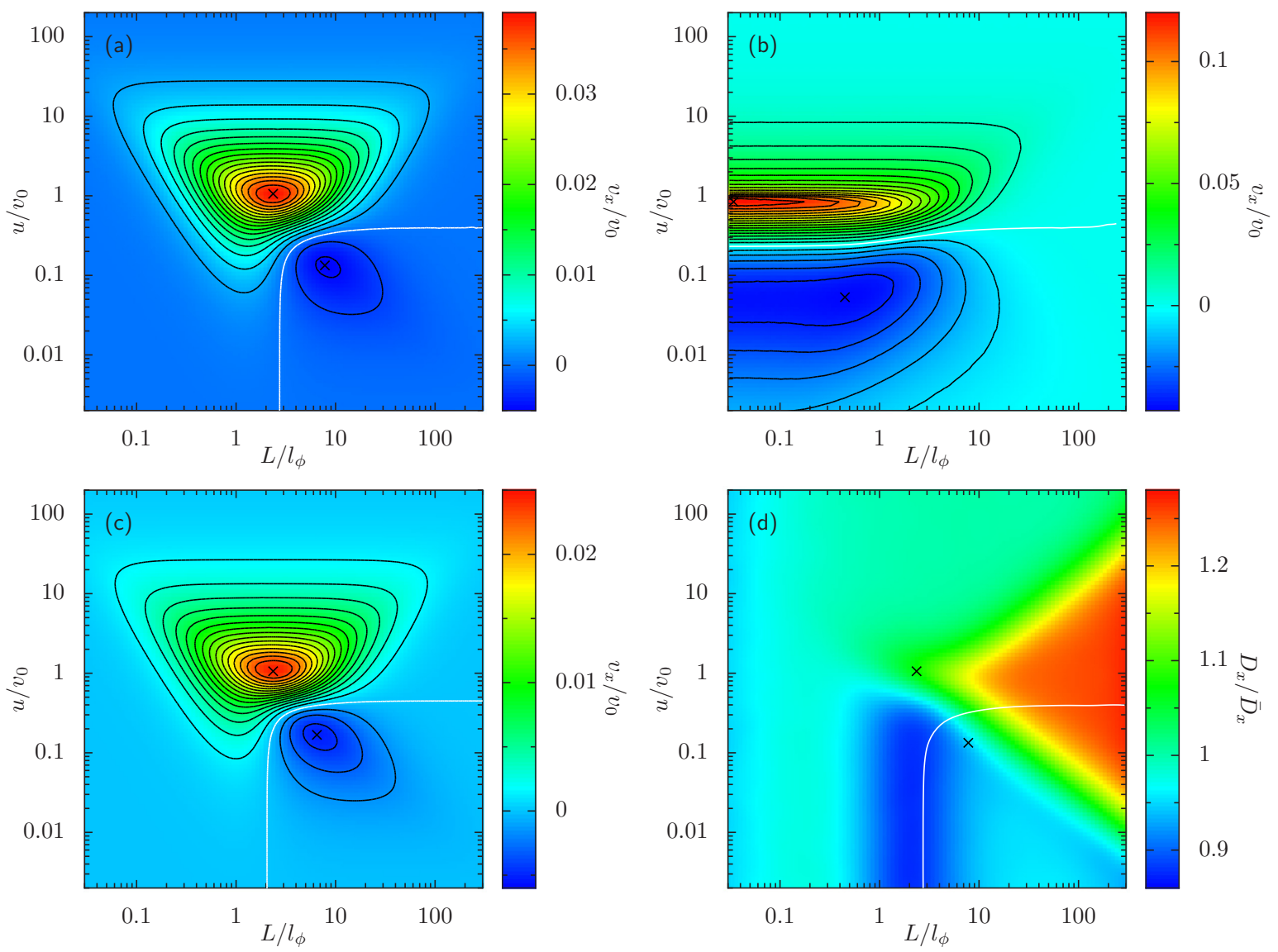

FIG. 2. Chemotaxis of an achiral swimmer with $\Omega=0$ in a sinusoidal traveling ADW. Unless differently stated, all model parameters were chosen consistently with reported experimental values [25]: $v_{0}=53 \mu \mathrm{m} / \mathrm{s}, D_{\phi}=165 \mathrm{~s}^{-1}$, and $D_{0}=2.2 \mu \mathrm{m}^{2} / \mathrm{s}$. The ADW waveform, $v(x)=w_{0}+\left(v_{0}-w_{0}\right) \sin ^{2}(\pi x / L)$, has fixed maxima, $v_{0}$, and tunable minima $w_{0}$, with $w_{0}<v_{0}$. All results are from numerical integration of the model Eqs. (1) or the corresponding FPE (7) (see Sec. IV). Lengths and velocities are given in units of $l_{\phi}$ and $v_{0}$, respectively. (a-c) Contour plots of the longitudinal drift velocity, $v_{x}=\lim _{t \rightarrow \infty}\langle x(t)-x(0)\rangle / t$ in the plane $(L, u) ; w_{0}=0$ and all other parameters are the same except for $D_{0}=0$ in panel (b) and $w_{0}=0.2$ in panel (c). The maxima of positive, $v_{x, \max }$, and negative, $v_{x, \min }$, chemotaxis are marked by black crosses; the separatrix curves, $u_{s}(L)$, delimiting the regions of positive and negative chemotaxis are drawn in white; (d) Contour plot of the diffusion constant $D_{x}=\lim _{t \rightarrow \infty}\left[\left\langle x^{2}\right\rangle-\langle x\rangle^{2}\right] / 2 t$ for the model parameters of panel (a). The scaling factor, $\bar{D}_{x}=D_{0}+v_{0}^{2} / 8 D_{\phi}$, is the swimmer's diffusion constant in the average velocity field $\langle v(x)\rangle=v_{0} / 2$. For animations of the swimmer's diffusion under diverse dynamical conditions, see Supplemental Material movies 3-6 [31].

longer distance to the right without bouncing against the right pulse wall. In the optimal case, $u \simeq v_{0}$, such a distance is of the order of the persistence length, $l_{\phi}$. Accordingly, for a fixed pulse width, $\Delta(\infty)$ attains a positive maximum around $u \simeq v_{0}$ and vanishes monotonically in the limit $u / v_{0} \rightarrow \infty$.

Consistent with our interpretation, in Figs. 1(c) and 1(d) the transition from negative to positive chemotaxis occurs when the time the particle takes to diffuse a length of the order of $l_{\phi}$ grows longer than the corresponding pulse crossing time, namely for $u / v_{0} \simeq 1 / 2$. Of course, under realistic experimental conditions, the apparent divergence of the shift, $\Delta(\infty) \rightarrow-\infty$, at vanishingly slow pulse speeds, Fig. 1(c), would be offset by the inevitable translational fluctuations with $D_{0}>0$, neglected in the simulations of Fig. 1. Moreover, for narrow pulses, $\sigma \lesssim l_{\phi}$, the swimmer cannot diffuse much inside the pulse, but crosses it ballistically. Consequently, as illustrated in Fig. 1(d), its positive drift is more pronounced than in the case $\sigma \gg l_{\phi}$. Viceversa, for large pulse widths the particle's dynamics is dominated by active diffusion and its displacement grows increasingly negative at small $u$. Of course, for $\sigma \rightarrow \infty$ the activant gradient becomes negligible and $\Delta(\infty)$ vanishes. Thus, in the regime of negative $\Delta(\infty)$, for any chosen $u$ there exists an optimal pulse width where the negative particle displacement is maximum.

\section{Chemotaxis by active density waves}

More generally, active pulses are generated in random or periodic sequences. For the sake of simplicity, we consider here the case of periodic ADWs with waveform

$$
v(x)=w_{0}+\left(v_{0}-w_{0}\right) \sin ^{2}(\pi x / L) .
$$

Like in most experimental setups, we assume that the parameters that regulate the swimmer's dynamics, $D_{0}, v_{0}, \tau_{\phi}$, and $l_{\phi}$, are fixed, whereas the ADW parameters, $u, L$, and $w_{0} / v_{0}$, can be tuned at the experimenter's convenience. We checked that the swimmer's chemotactic response is not appreciably 
modified by varying the sequence or waveform of the active pulses.

In the stationary regime, chemotaxis of achiral swimmers with $\Omega=0$ is characterized in terms of the drift velocity, $v_{x}=\langle\dot{x}\rangle$. This is plotted in Fig. 2 for different values of the wave parameters. The regions of the plane $(L, u)$ exhibiting positive or negative chemotaxis are delimited by separatrix curves, which depend also on $D_{0}$ and $w_{0}$ [Figs. 2(a)-2(c) and 3(b)]. Like in the case of single active pulses, negative chemotaxis is induced by slow ADWs only, with $u<v_{0}$ (Supplemental Material movies 3-6 [31]). Indeed, for fast ADWs, the distance a swimmer can travel without crossing a minimum of $v(x)$ is longer to the right than to the left. Hence, one can expect that $v_{x}>0$. Moreover, the distance the swimmer can surf a wave with $u \simeq v_{0}$ is limited solely by its persistence time, $\tau_{\phi}$, so that here positive chemotaxis is the most pronounced. Viceversa, for $u \ll v_{0}$, the swimmer crosses the ADW troughs, with reduced self-propulsion speed, both left and right. As the ADW propagates to the right, the time the swimmer takes to cross a trough to its left is shorter than to its right, hence $v_{x}<0$ (Supplemental Material movies 3 and 4 [31]). This argument certainly holds for $D_{0}=0$, where-provided $w_{0}=0$ - the particle can never cross a trough to the right, see Fig. 2(b) and Supplemental Material movies 4 and 6 [31].

The translational fluctuations, $D_{0}>0$, help the swimmer diffuse across the ADW troughs, suppressing the velocity rectification mechanism described above. Thus, with increasing $D_{0}$, the negative rectification effect becomes smaller compared to the positive surfing effect, until eventually $v_{x}$ changes sign. As the same effect cannot only be achieved by raising the noise strength $D_{0}$, but also by lowering the pulse periodicity $L$ (translational noise can easily "kick" particles out a narrow pulse), the separatrix bends downward, almost vertically, at a critical value of $L-$ see Fig. 2(a). The positive chemotaxis observed in the bottom-left quadrant of Fig. 2(a) is an unavoidable effect of the translational fluctuations $D_{0} \neq 0$.

The dependence of the vertical branch of the separatrix on the model parameters is illustrated by the curves of Figs. 3(b) and 3(c). We observe that its position along the horizontal axis is (i) shifted to the right proportional to $D_{0}$; (ii) shifted to the left proportional to $w_{0}$; and (iii) independent of $l_{\phi}$ (not shown). This behavior points to the existence of a critical value of $D_{0} / L v_{0},\left(D_{0} / L v_{0}\right)_{\mathrm{cr}}$, above which negative chemotaxis is suppressed. Moreover, Fig. 3(c) clearly shows that $\left(D_{0} / L v_{0}\right)_{\mathrm{cr}}$ grows linearly with $w_{0}$, independently of $l_{\phi}$. This implies that the vertical branch of the separatrix can be shifted to lower $L$ either by lowering $D_{0}$ or increasing $w_{0}$. However, these two options for enlarging the negative chemotaxis region of the $(L, u)$ plane have opposite impact on the modulus of $v_{x}$ chemotaxis is enhanced by lowering $D_{0}$ and suppressed by raising $w_{0}$ at constant $v_{0}$ [Fig. 3(c), inset].

The horizontal branch of the separatrix also depends on $w_{0}$, but is insensitive to the noise intensities $D_{0}$ and $D_{\phi}$. In the limit $D_{0} \rightarrow 0$, the separatrix is a smooth function of $L, u_{s}(L)$, with limits $u_{s}(0)$ and $u_{s}(\infty)$ of the same order of magnitude, both limits being functions of $w_{0}$ and smaller than $v_{0}$. In view of these results we conclude that negative chemotaxis is a robust property of the system, since it sets in under the most
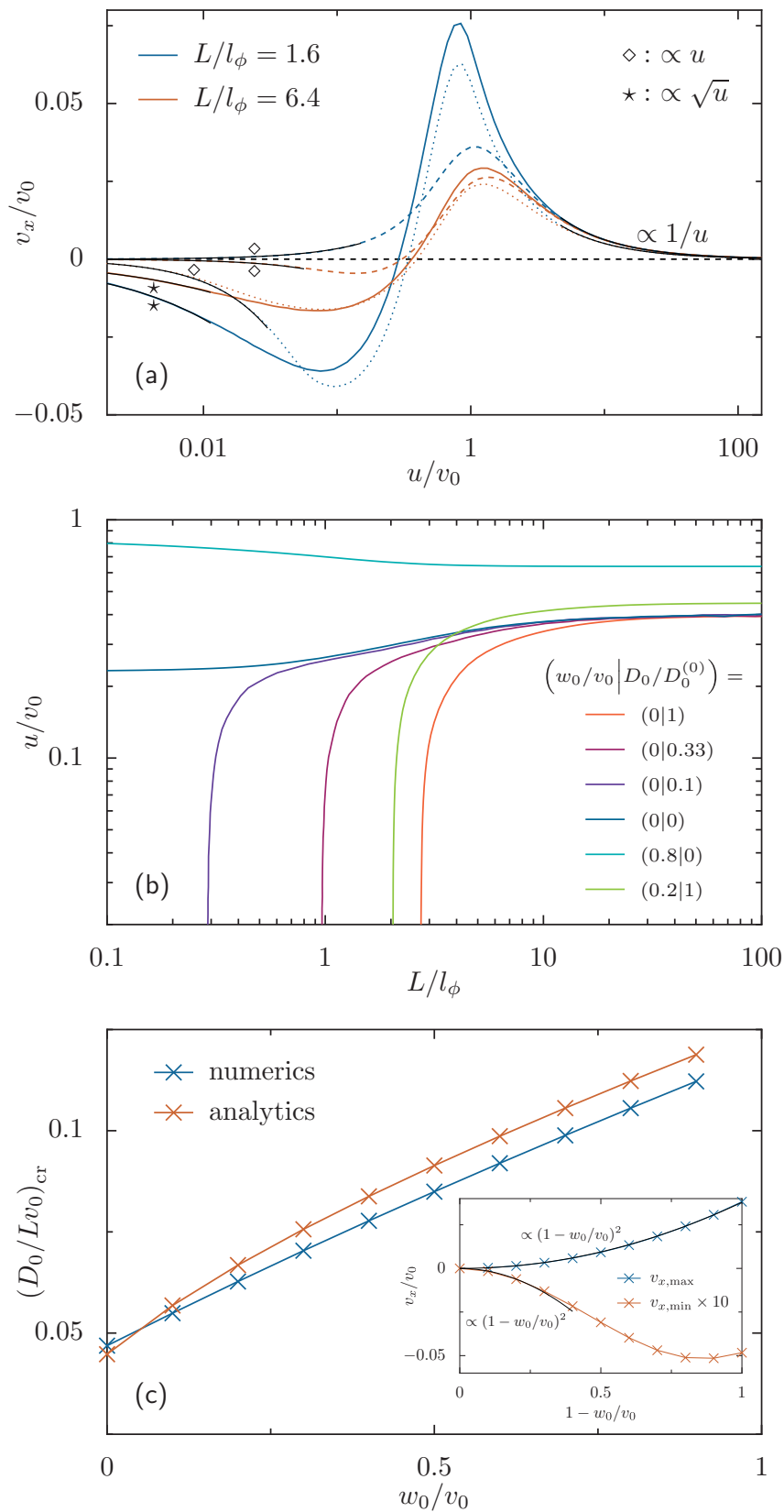

FIG. 3. Chemotaxis of an achiral swimmer in a sinusoidal traveling ADW. Unless differently stated, all parameters were chosen as in Fig. 2(a). Lengths and velocities are given in units of $l_{\phi}$ and $v_{0}$, respectively. (a) $v_{x}$ vs. $u$ in the ballistic regime, $L / l_{\phi}=1.6$, and diffusive regime, $L / l_{\phi}=6.4$, for finite $D_{0}$ and $w_{0}=0$ (dashed curves), $D_{0}=0$ and $w_{0}=0.1$ (dotted curves), and $D_{0}=0$ and $w_{0}=0$ (solid curves). All curves decay asymptotically with power laws, as indicated; (b) separatrix, $u_{s}$ vs. $L$ for different values of $D_{0}$ and $w_{0}$, where $D_{0}^{(0)}$ denotes the standard value of $2.2 \mu \mathrm{m}^{2} / \mathrm{s}$ used in the previous figures. The vertical branch of $u_{s}$ shifts to lower $L$ proportional to the noise level, $D_{0}$, and inversely proportional to the ADW baseline, $w_{0}$; (c) $\left(D_{0} / L v_{0}\right)_{\text {cr }}$ vs. $w_{0}$ from the numerical integration of the exact Eq. (7) (blue crosses), and the approximated FPE in the ballistic regime (red crosses); see Eq. (11). In the inset the maxima and minima of $v_{x}, v_{x, \max }$, and $v_{x, \min }$, are shown to increase faster than linearly with the amplitude of the ADW, $v_{0}-w_{0}$. Note, however, that the modulus of $v_{x, \min }$ increases with $w_{0}$ for $w_{0} \ll v_{0}$, as also shown in panel (c). 

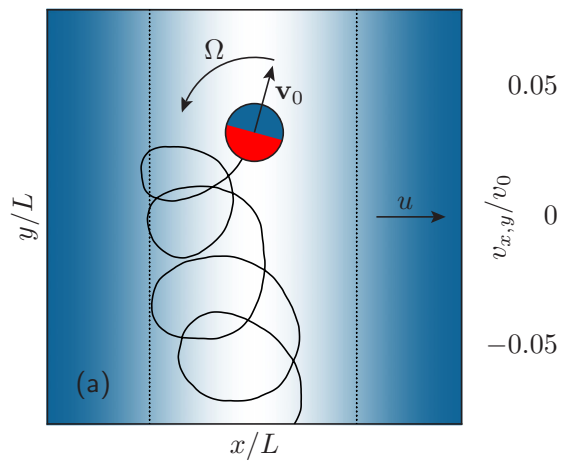

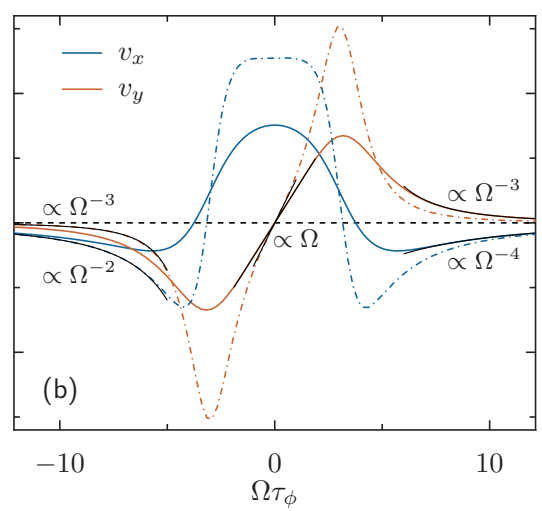

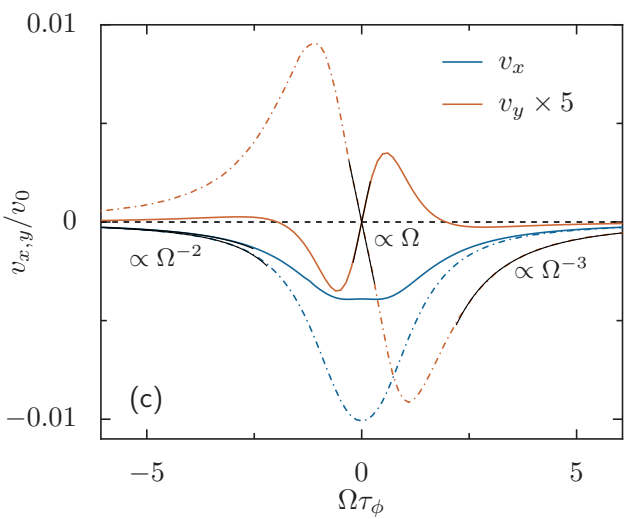

FIG. 4. Chemotaxis of a chiral swimmer with $\Omega \neq 0$ in a sinusoidal traveling ADW. Unless differently stated, all model parameters were chosen as in Fig. 2(a). All results are from numerical integration of the model Eqs. (1) or the corresponding FPE (7). The drift velocities and chiral frequency are given in units of $v_{0}$ and $D_{\phi}=\tau_{\phi}^{-1}$, respectively. For animations see Supplemental Material movies 7-10 [31]. In panel (a) is a sketch of the upward spiraling trajectory of a swimmer bouncing against the left trough of an ADW, where the dotted black line indicates the half-width. The longitudinal and transverse drift velocities, $v_{x}=\lim _{t \rightarrow \infty}\langle x(t)-x(0)\rangle / t$ and $v_{y}=\lim _{t \rightarrow \infty}\langle y(t)-y(0)\rangle / t$ are plotted vs. $\Omega \tau_{\phi}$ for (b) $L / l_{\phi}=2, u / v_{0}=1$; and (c) $L / l_{\phi}=7, u / v_{0}=0.2$ (solid curves). The corresponding curves for $D_{0}=0$ are also drawn for the sake of comparison (dashed curves). The exponents of fitted power-laws coincide with those predicted in Sec. III.

affordable experimental conditions of ADWs traveling with low speed, $u \ll v_{0}$, and long wavelength, $L \gg l_{\phi}$.

\section{Transverse chemotaxis of chiral swimmers}

An intrinsic rotational torque of the swimmer can be either the accidental result of fabrication defects or the desired effect obtained, e.g., by bending an active nanorod [32]. In any case, chirality strongly impacts swimmers' chemotaxis. In Figs. 4(b) and 4(c) the longitudinal and transversal drift velocities, $v_{x}=$ $\langle\dot{x}\rangle$ and $v_{y}=\langle\dot{y}\rangle$, are plotted against the torque frequency, $\Omega$, for conditions of, respectively, the largest positive and negative chemotaxis at $\Omega=0$ [marked by crosses in the contour plot of Fig. 2(a)]. A few remarkable properties are immediately apparent (see also Supplemental Material movies 7-10 [31]): (i) chirality induces a transverse chemotactic drift, $v_{y} \neq 0$. Such an effect is the strongest in the regime of positive longitudinal chemotaxis of achiral particles; (ii) $v_{x}(\Omega)$ and $v_{y}(\Omega)$ are, respectively, even and odd functions of $\Omega$, consistent with the symmetry of the model Eqs. (1) under the transformation $\phi \rightarrow-\phi$; (iii) chirality tends to suppress longitudinal chemotaxis. This effect is best noticeable in Fig. 4(b), where for $\Omega \tau_{\phi} \sim \pi$ the longitudinal drift velocity, $v_{x}$, drops to zero, while $v_{y}$ develops a peak of height comparable with $v_{x}(0)$. Under these conditions, the chemotactic effect of the incoming ADW is fully transverse and with the same sign as $\Omega$. At even higher frequencies, $v_{x}$ changes sign from positive to negative; (iv) in the regime of negative chemotaxis of achiral particles and zero translational noise [see Fig. 2(b)], the curves $v_{x}(\Omega)$ and $v_{y}(\Omega)$ have the same sign and no zeros for $\Omega>0$. On raising $D_{0}$, without changing the sign of $v_{x}$, transverse chemotaxis is suppressed and its sign varies with increasing $\Omega$.

The mechanism responsible for transverse chemotaxis is illustrated in Fig. 4(a). An active particle subject to a positive torque, $\Omega>0$, bounces against the ADW troughs, thus tracing spiraling circles of radius $r_{d} \sim v_{0} / \Omega$, which go up (down) the right-hand (left-hand) side of the trough. For fast-traveling ADWs with $u>v_{0}$, bouncing trajectories only take place on the right-hand side of the wave troughs, so that $v_{y}>0$. Of course, transverse chemotaxis is most pronounced when the bouncing process is synchronized with the wave modulation, namely, when the bouncing time, $\pi / \Omega$, is of the order of the wave period, $L / u$. Moreover, such a mechanism grows more effective for swimmer persistence times larger than the bouncing times, that is, for $\Omega \tau_{\phi} \gtrsim \pi$. Both conditions are satisfied in Fig. 4(b), so that, in an appropriate $\Omega \tau_{\phi}$ range, transverse chemotaxis supersedes longitudinal chemotaxis.

For slow ADWs, spiraling trajectories develop on both sides of the wave minima. As noticed above, trough crossings from right to left take a shorter time than from left to right; accordingly, upward bouncing trajectory arcs have shorter span than the downward ones. This observation explains why, in Fig. 4(c) for $u \ll v_{0}$ and $D_{0}=0$, transverse chemotaxis and chirality have opposite signs. Also, similar to achiral chemotaxis, translational noise eases ADW trough crossings in both directions, thus suppressing transverse chemotaxis and eventually reversing its orientation. It is also suppressed by reducing the ADW amplitude.

\section{DISCUSSION}

We next present a more quantitative analysis of our results based on the approximation schemes detailed in Sec. IV.

\section{A. Ballistic regime}

The curves of the drift velocity, $v_{x}$, versus the ADW speed, $u$, in Fig. 3(a) exhibit a characteristic resonant behavior for both positive and negative chemotaxis. In the ballistic regime, i.e., for $L \ll l_{\phi}$, their decay is satisfactorily described in the two-state approximation, where, for sufficiently long persistence times (compared to the pulse crossing time), the swimmer's dynamics is modeled as the superposition of a nonfluctuating, self-propelling drift to the right and to the left. In the absence of translational noise, the predicted average drift velocity, Eq. (5), decays like: (i) $v_{x} / v_{0} \propto v_{0} / u$ for $u \gg v_{0}$ (to hold for any value of $L / l_{\phi}$ ); (ii) $v_{x} / v_{0} \propto-u / v_{0}$ for 
$u \ll v_{0}$ and $w_{0}>0$; and (iii) $v_{x} / v_{0} \propto-\sqrt{u / v_{0}}$ for $u \ll v_{0}$ and $w_{0}=0$. These predictions closely agree with the numerical fits of Fig. 3(a). In addition, Eq. (5) suggests that the positive chemotaxis maxima, $v_{x, \max }$, occur for $u \simeq v_{0}$, as expected, and the negative minima, $v_{x, \max }$, for $u \lesssim w_{0}$. The two-state model also provides a close estimate of the horizontal branch of the separatrix, $u_{s}(L)$. The limit $L / l_{\phi} \rightarrow 0$ for $D_{0}=0, u_{s}(0)$, can be approximated analytically by setting $v_{x}=0$ in the second line of Eq. (5) and solving for $u$. Hence, $u_{s}(0) / v_{0}=$ $\left[\left(w_{0}+v_{0}\right) / 6 v_{0}\right]\left[1+\sqrt{1+12 v_{0} w_{0} /\left(v_{0}+w_{0}\right)^{2}}\right]$, in quantitative agreement with the numerical data of Fig. 3(b). The full curves $u_{s}(L)$ plotted there were obtained by locating the zeros of the average current, Eq. (9), as a function of $u$ and $L$.

As remarked in Sec. II, translational fluctuations tend to suppress chemotaxis at large and negative chemotaxis in particular. In the two-state model notation, this is a consequence of the noise-induced "creeping effect" mentioned in Sec. IV, an effect more conveniently addressed in the FPE formalism. For instance, upon expanding the ballistic approximation of the average current, Eq. (11), in powers of $u / v_{0}$, the chemotactic speed is proven to grow proportionally to $u$ (and not $\sqrt{u}$ ) even at $w_{0}=0$, with a sign that turns from negative to positive on increasing $D_{0}$ [Fig. 3(a)]. More interestingly, Eq. (11) allowed us to locate the vertical separatrix branch of the contour plots of Figs. 2(a)-2(c), namely the quantity $\left(D_{0} / L v_{0}\right)_{\text {cr }}$ plotted in Fig. 3(c). For an analytical estimate of the same quantity, we notice that translational noise provides an additional diffusion mechanism that competes with self-propulsion. The self-propulsion mechanism eventually prevails when the time the particle takes to diffuse a half ADW wavelength due to translational noise, $(L / 2)^{2} / 2 D_{0}$, is shorter than the average time to cross the same distance with an average speed $\left(v_{0}+w_{0}\right) / 2$ in the ballistic regime [see also Eq. (5)], $2 L /\left(v_{0}+w_{0}\right)$. This occurs for $D_{0} / L v_{0} \gtrsim\left(D_{0} / L v_{0}\right)_{\mathrm{cr}}$, with $\left(D_{0} / L v_{0}\right)_{\text {cr }} \propto 1+w_{0} / v_{0}$, in agreement with the numerical data in Fig. 3(c). We remarked in Sec. II that $\left(D_{0} / L v_{0}\right)_{\mathrm{cr}}$ is independent of $\tau_{\phi}$; therefore, this analytical estimate holds in the ballistic and diffusive regimes, alike.

Contrary to the Stokes drift [16], $v_{x}$ at small $u$ grows with exponent clearly smaller than 2 , irrespective of $w_{0}$ and $D_{0}$ (and, therefore, of its own sign): this means that the chemotactic effect studied here is not governed by the fore-rear ADW gradients so much as by the swimmer's diffusion across the traveling ADW troughs.

\section{B. Diffusive regime}

In the diffusive regime, i.e., for $L \gg l_{\phi}$, the persistence time $\tau_{\phi}$ can be taken as vanishingly small. Accordingly, the swimmer's diffusion in the wave direction is closely described by the multiplicative LE in Eq. (6). Standard Stratonovitch calculus $[33,34]$ yields the finite drift term,

$$
v_{x}=\left[\left(v_{0}-w_{0}\right)^{2} / 4 D_{\phi}\right]\left\langle(d / d x) \sin ^{2}[\pi(x-u t) / L]\right\rangle,
$$

where $\langle\ldots\rangle$ denotes a stationary average over the stochastic trajectories of $x(t)$. This average was computed explicitly by solving the corresponding FPE, Eq. (12): for $u<v_{0}$ chemotaxis indeed turns out to be negative with $v_{x} \propto-u$ for $D_{0}>0$ and $u \ll v_{0}$, and $v_{x} \propto-\sqrt{u}$ for $D_{0}=0$ and $u \ll v_{0}$, as in the fits of Fig. 3(a).
On the other hand, upon increasing $u$ larger than the modulus of the multiplicative term in Eq. (6), $u>|\langle v(x) \cos \phi\rangle| \simeq$ $\left(w_{0}+v_{0}\right) / 2 \sqrt{2}$, the dynamical effect of the angular fluctuations is suppressed with respect to the dragging action of the ADW, so that chemotaxis changes sign from negative to positive. The right-hand side of the above inequality approximates the horizontal asymptote of $u_{s}(L)$ in the limit $L / l_{\phi} \rightarrow \infty$. As displayed in Fig. 3(b), $u_{s}(\infty)$ is a function of $w_{0}$, only.

The role of the separatrix is further illustrated by the contour plot of Fig. 2(d), where we plotted the diffusion constant $D_{x}=\lim _{t \rightarrow \infty}\left[\left\langle x^{2}\right\rangle-\langle x\rangle^{2}\right] / 2 t$ for the model parameters of Fig. 2(a). The computed values of $D_{x}$ have been compared with the swimmer's average diffusion constant, $\bar{D}_{x}$, defined in the figure caption. One sees immediately that $D_{x} / \bar{D}_{x} \simeq 1$ almost everywhere in the $(L, u)$ plane, except across the (hot) horizontal and (cold) vertical branches of the separatrix, where $D_{x} / \bar{D}_{x}>1$ and $D_{x} / \bar{D}_{x}<1$, respectively. Indeed, the chemotaxis sign inversion observed upon increasing the ADW speed, $u$, signals a locked-running transition in the two-state model Eqs. (3), a mechanism known to produce excess diffusion [35-37]. On the other hand, the chemotaxis inversion obtained by increasing the ADW wavelength is governed by translational noise (it never occurs for $D_{0}=0$ ), which acts there as a sort of lubricant, thus suppressing the net swimmer diffusivity. With a view to experimental demonstration, we remark that for artificial swimmers, chemotaxis by traveling ADWs is no more dispersive than regular transport in the bulk.

\section{Chiral chemotaxis}

The FPE formalism also allows a better characterization of chiral chemotaxis. To shed light on the underlying irreducible 2D mechanism we considered the model Eqs. (1) in the opposite adiabatic limits $\Omega \tau_{\phi} \ll 1$ and $\Omega \tau_{\phi} \gg 1$, both in the ballistic and diffusive regimes introduced above. A systematic perturbation approach (not reported here) led us to conclude that $v_{x}=c_{1}\left(v_{0}-w_{0}\right)$ and $v_{y}=s_{1}\left(v_{0}-w_{0}\right)$, where $c_{1}$ and $s_{1}$ are, respectively, even and odd functions of $\Omega$. In particular, for $\Omega \tau_{\phi} \ll 1$ we obtained $c_{1} \propto \Omega^{0}$ and $s_{1} \propto \Omega$ for any $D_{0}$; for $\Omega \tau_{\phi} \gg 1, c_{1}$ decays like $\Omega^{-2}$ at $D_{0}=0$ and $\Omega^{-4}$ at $D_{0} \neq 0$, whereas $s_{1} \propto \Omega^{-3}$, independent of the translational noise strength $D_{0}$.

We conclude by underscoring that chemotaxis of artificial microswimmers is a robust phenomenon that lends itself to accessible laboratory demonstrations and promising applications to nanotechnology and medical sciences. Our numerical results and interpretation go beyond the earlier "chemotactic wave paradox" debate, insofar as the swimmer's tactic response results from its ability of diffusing within a traveling active pulse and not just the asymmetry of the fore-rear pulse gradients. Moreover, the direction and magnitude of the tactic response are extremely sensitive to the self-propulsion mechanism, which suggests the design of tactic devices to control the production and transport of artificial swimmers.

\section{METHODS}

The model Eqs. (1) describe the spatial diffusion of an active over-damped Brownian particle subject to a traveling field of 
force $v(x, t)$. Contrary to the previous literature on Brownian Stokes' drift $[16,17]$, here the particle's motion is characterized by a finite persistence time, $\tau_{\phi}$, and related length $l_{\phi}=v_{0} \tau_{\phi}$. A simple calculation [38] shows that the angular factors, $\cos \phi$ and $\sin \phi$, decay exponentially, $\langle\cos \phi(t) \cos \phi(0)\rangle=$ $\langle\sin \phi(t) \sin \phi(0)\rangle=(1 / 2) \exp \left(-D_{\phi}|t|\right)$, hence $\tau_{\phi}=1 / D_{\phi}$. Persistence (or memory effects) become appreciable when the Brownian particle is confined to geometries with characteristic size smaller than $l_{\phi}$. In our model, the standard distinction between a ballistic regime with $L \ll l_{\phi}$ and a diffusive regime with $L \gg l_{\phi}$ must be revised to account for the finite wave period $L / u$, as discussed below.

\section{A. The Langevin equations}

The stochastic differential Eqs. (1) were numerically integrated by means of a standard Euler-Maruyama scheme [39]. The stochastic averages were taken over an ensemble of trajectories with random initial swimmer orientation, $\phi(0) \in$ $[0,2 \pi]$.

\section{The two-state model}

In the ballistic regime, the analytic treatment of Eqs. (1) with $\Omega=0$ is greatly simplified by assuming that an achiral swimmer moves in the wave's direction with equal probability to the right, $\phi=0$, or to the left, $\phi=\pi$, i.e., with velocity $\pm v(x, t)$, thus totally ignoring its transverse motion along $y$. The ensuing swimmer dynamics is modeled through two independent LEs,

$$
\dot{x}= \pm w_{0} \pm\left(v_{0}-w_{0}\right) \sin ^{2}[\pi(x-u t) / L]+\sqrt{D_{0}} \xi_{x}(t)
$$

obtained by setting $\cos \phi= \pm 1$ in Eq. (1). By introducing the auxiliary variable $x^{\prime}=x-u t$, Eqs. (3) can rewritten in the form of two LEs for for a tilted potential with different tilting,

$$
\begin{aligned}
\dot{x}^{\prime}= & -u \pm\left[\left(v_{0}+w_{0}\right)-\left(v_{0}-w_{0}\right) \cos \left(2 \pi x^{\prime} / L\right)\right] / 2 \\
& +\sqrt{D_{0}} \xi_{x}(t)
\end{aligned}
$$

The time a noiseless swimmer takes to cross a wavelength $L$ can be calculated analytically from Eqs. (4) with $D_{0}=0$. In the steady-state with $\phi=0$ (oriented to the right), we obtained $\dot{x}^{\prime}>0$ for $0 \leqslant u \leqslant w_{0}, \dot{x}^{\prime}=0$ for $w_{0} \leqslant u \leqslant v_{0}$, and $\dot{x}^{\prime}<0$ for $u \geqslant v_{0}$; in the steady-state with $\phi=\pi$ (oriented to the left), $\dot{x}^{\prime}<0$ for all $u$. The coordinate $x^{\prime}$ is thus locked for $\phi=0$ and $w_{0} \leqslant u \leqslant v_{0}$, and running under all remaining conditions, with $\left|v_{x^{\prime}}^{( \pm)}\right|=L / t_{ \pm}$for $\phi=0$ and $\phi=\pi$, respectively, where $t_{ \pm}=L / \sqrt{\left(v_{0} \mp u\right)\left(w_{0} \mp u\right)}$. Accordingly, on transforming back to the coordinate $x$, we obtain two distinct solutions for $\langle\dot{x}\rangle, v_{x}^{(+)}$and $v_{x}^{(-)}$. Finally, averaging over the two $\phi$ states corresponds to taking the arithmetic mean $v_{x}=\left[v_{x}^{(+)}+v_{x}^{(-)}\right] / 2$, that is

$$
v_{x}=\left\{\begin{array}{l}
u+\frac{1}{2}\left[\sqrt{\left(v_{0}-u\right)\left(w_{0}-u\right)}-\sqrt{\left(v_{0}+u\right)\left(w_{0}+u\right)}\right] \\
0 \leqslant u \leqslant w_{0} \\
u-\frac{1}{2} \sqrt{\left(v_{0}+u\right)\left(w_{0}+u\right)} \quad w_{0} \leqslant u \leqslant v_{0} \\
u-\frac{1}{2}\left[\sqrt{\left(u-v_{0}\right)\left(u-w_{0}\right)}+\sqrt{\left(u+v_{0}\right)\left(u+w_{0}\right)}\right] \\
u \geqslant v_{0}
\end{array} .\right.
$$

As shown in Sec. III, despite the rather rough assumptions detailed above, this result proves to be an effective interpretation tool.

Such a ballistic scheme holds in a strict sense under the condition that $\tau_{\phi}>\max \left\{t_{+}, t_{-}\right\}$, namely for $u / v_{0}>$ $\left[\sqrt{\left(2 L / l_{\phi}\right)^{2}+\left(1-w_{0} / v_{0}\right)^{2}}+\left(1+w_{0} / v_{0}\right)\right] / 2$. This implies that Eq. (5) applies for $u>v_{0}$ when $L / l_{\phi} \ll 1$, and for $u / v_{0}>L / l_{\phi}$ when $L / l_{\phi} \gg 1$. Moreover, since in the state $\phi=0$ with $w_{0}<u<v_{0}$ the particle is locked, i.e., $t_{+}=\infty$, the solution for $v_{x}$ holds approximately under the weaker condition $\tau_{\phi}>t_{-}$. This is why in Sec. III we extended our two-state model interpretation to $u \ll v_{0}$ and $L \ll l_{\phi}$.

To solve the two-state model of Eqs. (3) in the presence of translational noise we had recourse to the FPE formalism, as discussed below. We anticipate that for $D_{0}>0$ one obtains $v_{x^{\prime}}^{(+)}>0$ also in the range $w_{0} \leqslant u \leqslant v_{0}$ [34]: translational noise smooths the locked-running transition, thus acting as a dynamical lubricant (creeping effect).

\section{1D reduced model}

Brownian motion can be treated as purely diffusive when its persistence length is much shorter than all other length scales of the system. For the swimmer of Eq. (1) this condition amounts to requiring that the persistence time, $\tau_{\phi}$, is shorter than both crossing times $t_{+}$and $t_{-}$, namely for $u / v_{0} \leqslant\left[\sqrt{\left(2 L / l_{\phi}\right)^{2}-\left(1-w_{0} / v_{0}\right)^{2}}-\left(1+w_{0} / v_{0}\right)\right] / 2$. In the limit of vanishingly small $\tau_{\phi}$, the random orientation factor in $\mathrm{Eq}$. (1), $\cos \phi(t)$, can be replaced by an effective noise source with zero mean and autocorrelation function $\langle\cos \phi(t) \cos \phi(0)\rangle \simeq 2 \delta(t) /\left(2 D_{\phi}\right)$ [38], so that the dynamics of $x^{\prime}=x-u t$ is well described by the effective 1D LE,

$$
\dot{x}^{\prime}=-u+v\left(x^{\prime}\right) \xi_{\phi}(t) / D_{\phi} \sqrt{2}+\sqrt{D_{0}} \xi_{x}(t)
$$

where the multiplicative stochastic term on the r.h.s. must be interpreted in Stratonovitch sense [33,40].

\section{B. The Fokker-Planck formalism}

For a more detailed analysis of the swimmer's stochastic dynamics we turn to the Fokker-Planck equation (FPE) associated with the model Eqs. (1) [33,34],

$$
\begin{aligned}
\partial_{t} P(\mathbf{r}, \phi, t) & =-\nabla_{i} J_{i}(\mathbf{r}, \phi, t), \\
\mathbf{J}(\mathbf{r}, \phi, t) & =\left(\begin{array}{c}
-D_{0} \partial_{x}+v(x) \cos \phi-u \\
-D_{0} \partial_{y}+v(x) \sin \phi \\
-D_{\phi} \partial_{\phi}+\Omega
\end{array}\right) P(\mathbf{r}, \phi, t),
\end{aligned}
$$

and $\nabla=\left(\partial_{x}, \partial_{y}, \partial_{\phi}\right)$. Here, $\mathbf{r}=(x, y)$ denotes the particle's spatial coordinates in the ADW moving frame, $x-u t \rightarrow x$ and $y \rightarrow y$ [the prime sign used in Eq. (4) has been dropped for simplicity]. The 3D functions $P$ and $\mathbf{J}$ denote, respectively, the probability density and current of the particle in the state $(\mathbf{r}, \phi)$ at time $t$. The FPE (7) was numerically integrated by combining the method of lines [41] with a secondorder backward-difference scheme [42]. Periodic boundary conditions were assumed for all three variables $x, y$, and $\phi$, with relevant periods $L, L$, and $2 \pi$. This amounts to the 
one-zone reduced formulation of probability density [43],

$$
\hat{P}(\mathbf{r}, \phi, t)=\sum_{k, n, m=-\infty}^{\infty} P(x+k L, y+n L, \phi+2 \pi m, t) .
$$

The particle's drift velocities in the ADW frame are then computed as the stationary currents in the $x$ and $y$ direction,

$$
v_{x, y}=\int_{0}^{L} d x \int_{0}^{L} d y \int_{0}^{2 \pi} d \phi \lim _{t \rightarrow \infty} J_{x, y}(\mathbf{r}, \phi, t) .
$$

The final result for $v_{x}$ is obtained by transforming it back to the laboratory frame, $v_{x} \rightarrow v_{x}+u$. For achiral particles, $\Omega=0$, the transverse coordinate $y$ is dispensable, so that, upon integration over $y$, the FPE (7) is immediately reduced to a partial differential equation for the $2 \mathrm{D}$ probability density $P(x, \phi, t)$. In the following we shortly address two limiting cases of such a reduced FPE, corresponding, respectively, to the two-state model and the reduced 1D model approximations of the LEs Eq. (1) with $\Omega=0$, as introduced above.

\section{Ballistic approximation}

In the ballistic regime, the particle's orientation is almost constant during its spatial relaxation. Accordingly, the FPE can be further reduced to a 1D partial differential equation in $x$ for a fixed but arbitrary $\phi$. The stationary reduced probability current has a manageable expression as a function of $\phi$ [43], i.e.,

$$
\begin{aligned}
\hat{J}(\phi)= & \frac{D_{0}}{L^{2}}\left\{1-\exp \left[-\frac{L v_{0}}{D_{0}}\left(\frac{v_{0}+w_{0}}{2 v_{0}} \cos \phi-\frac{u}{v_{0}}\right)\right]\right\}\left\{\int _ { 0 } ^ { 1 } d \xi \int _ { 0 } ^ { 1 } d \zeta \operatorname { e x p } \left[-\frac{L v_{0}}{D_{0}}\left(\left(\frac{v_{0}+w_{0}}{2 v_{0}} \cos \phi-\frac{u}{v_{0}}\right) \zeta\right.\right.\right. \\
& \left.\left.\left.-\frac{v_{0}-w_{0}}{4 \pi v_{0}} \cos \phi\{\sin [2 \pi(\zeta+\xi)]-\sin (2 \pi \xi)\}\right)\right]\right\}^{-1},
\end{aligned}
$$

which, upon averaging over a uniform $\phi$ distribution with $\phi \in[0,2 \pi]$ and transforming back to the laboratory frame, yields the most accurate estimates of the drift velocity in the ballistic regime,

$$
v_{x}=\frac{L}{2 \pi} \int_{0}^{2 \pi} \mathrm{d} \phi \hat{J}(\phi)+u .
$$

\section{Diffusive approximation}

In the opposite limit of fast angular relaxation, the dependence on the coordinate $\phi$ can be projected out by means of a mapping procedure [44] to be detailed in an upcoming technical report. In leading order of the perturbation parameter $l_{\phi} / L$, we obtained a $1 \mathrm{D}$ partial differential equation,

$$
\partial_{t} P(x, t)=\partial_{x}^{2} a(x) P(x, t)-\partial_{x} b(x) P(x, t),
$$

with $\quad a(x)=v^{2}(x) / 2 D_{\phi}+D_{0} \quad$ and $\quad b(x)=(d / d x) v^{2}(x) /$ $4 D_{\phi}-u$. This is the FPE corresponding to the reduced multiplicative process derived in Eq. (6). Upon computing the stationary probability current,

$$
\begin{aligned}
\hat{J}= & {\left[1-\exp \left(-\int_{0}^{L} d x \frac{b(x)}{a(x)}\right)\right] } \\
& \times\left[\int_{0}^{L} d x \int_{0}^{L} d y \frac{1}{a(x)} \exp \left(\int_{x}^{y+x} d z \frac{b(z)}{a(z)}\right)\right]^{-1},
\end{aligned}
$$

the drift velocity is finally expressed as $v_{x}=L \hat{J}+u$.

\section{ACKNOWLEDGMENTS}

This work has been supported by the cluster of excellence Nanosystems Initiative Munich (P.H.). P.H. and F.M. acknowledge financial support from the Center for Innovative Technology (ACIT) of the University of Augsburg. S.S. thanks the Alexander von Humboldt Stiftung for support through a Friedrich W. Bessel Research Award.
[1] J. D. Murray, Mathematical Biology (Springer, Berlin, 1989).

[2] H. C. Berg, E. coli in Motion (Springer, New York, 2004).

[3] J. Elgeti, R. G. Winkler, and G. Gompper, Physics of microswimmers-Single particle motion and collective behavior: A review, Rep. Progr. Phys. 78, 056601 (2015).

[4] F. Schweitzer, Brownian Agents and Active Particles (Springer, Berlin, 2003).

[5] S. Jiang and S. Granick (Eds.), Janus Particle Synthesis, Self-Assembly and Applications (RSC Publishing, Cambridge, 2012); A. Walther and A. H. E. Müller, Janus particles: Synthesis, self-assembly, physical properties, and applications, Chem. Rev. 113, 5194 (2013).

[6] Y. Hong, D. Velegol, N. Chaturvedi, and A. Sen, Biomimetic behavior of synthetic particles: From microscopic randomness to macroscopic control, Phys. Chem. Chem. Phys. 12, 1423 (2010)

[7] Y. Hong, N. M. K. Blackman, N. D. Kopp, A. Sen, and D. Velegol, Chemotaxis of Nonbiological Colloidal Rods, Phys. Rev. Lett. 99, 178103 (2007).

[8] H.-R. Jiang, N. Yoshinaga, and M. Sano, Active Motion of a Janus Particle by Self-Thermophoresis in a Defocused Laser Beam, Phys. Rev. Lett. 105, 268302 (2010).

[9] L. Baraban, S. M. Harazim, S. Sanchez, and O. G. Schmidt, Chemotactic behavior of catalytic motors in microfluidic channels, Angew. Chem. Int. Ed. 52, 5552 (2013).

[10] J.-X. Chen, Y.-G. Chen, and Y.-Q. Ma, Chemotactic dynamics of catalytic dimer nanomotors, Soft Matter 12, 1876 (2016). 
[11] G. H. Wadhams and J. P. Armitage, Making sense of it all: Bacterial chemotaxis, Nat. Rev. Mol. Cell Biol. 5, 1024 (2004).

[12] P. Hänggi and F. Marchesoni, Artificial Brownian motors: Controlling transport on the nanoscale, Rev. Mod. Phys. 81, 387 (2009).

[13] P. S. Burada, P. Hänggi, F. Marchesoni, G. Schmid, and P. Talkner, Diffusion in confined geometries, ChemPhysChem 10, 45 (2009).

[14] P. R. Fisher, R. Merkl, and G. Gerisch, Quantitative analysis of cell motility and chemotaxis in Dictyostelium discoideum by using an image processing system and a novel chemotaxis chamber providing stationary chemical gradients, J. Cell Biol. 108, 973 (1989).

[15] G. G. Stokes, On the theory of oscillatory waves, Trans. Camb. Phil. Soc. 8, 441 (1849).

[16] C. Van den Broeck, Stokes' drift: An exact result, Europhys. Lett. 46, 1 (1999).

[17] M. Borromeo and F. Marchesoni, Brownian surfers, Phys. Lett. A 249, 199 (1998).

[18] T. Höfer, P. K. Maini, J. A. Sherratt, M. A. J. Chaplain, P. Chauvet, D. Metevier, P. C. Montes, and J. D. Murray, A resolution of the chemotactic wave paradox, Appl. Math. Lett. 7, 1 (1994).

[19] S. Thakur, J.-X. Chen, and R. Kapral, Interaction of a chemically propelled nanomotor with a chemical wave, Angew. Chem. Int. Ed. 50, 10165 (2011).

[20] S. van Teeffelen and H. Löwen, Dynamics of a Brownian circle swimmer, Phys. Rev. E 78, 020101 (2008).

[21] B. ten Hagen, F. Kümmel, R. Wittkowsky, D. Takagi, H. Löwen, and C. Bechinger, Gravitaxis of asymmetric self-propelled colloidal particles, Nature Commun. 5, 4829 (2014).

[22] B. ten Hagen, R. Wittkowski, D. Takagi, F. Kümmel, C. Bechinger, and H. Löwen, Can the self-propulsion of anisotropic microswimmers be described by using forces and torques?, J. Phys.: Condens. Matter 27, 194110 (2015).

[23] X. Ao, P. K. Ghosh, Y. Li, G. Schmid, P. Hänggi, and F. Marchesoni, Active Brownian motion in a narrow channel, Eur. Phys. J.- Special Topics 223, 3227 (2014).

[24] I. Buttinoni, G. Volpe, F. Kümmel, G. Volpe, and C. Bechinger, Active Brownian motion tunable by light, J. Phys.: Condens. Matter 24, 284129 (2012).

[25] G. Volpe, I. Buttinoni, D. Vogt, H.-J. Kümmerer, and C. Bechinger, Microswimmers in patterned environments, Soft Matter 7, 8810 (2013).

[26] P. K. Ghosh, Y. Li, F. Marchesoni, and F. Nori, Pseudochemotactic drifts of artificial microswimmers, Phys. Rev. E 92, 012114 (2015).

[27] J. R. Howse, R. A. L. Jones, A. J. Ryan, T. Gough, R. Vafabakhsh, and R. Golestanian, Self-Motile Colloidal Particles:
From Directed Propulsion to Random Walk, Phys. Rev. Lett. 99, 048102 (2007).

[28] M. J. Schnitzer, Theory of continuum random walks and application to chemotaxis, Phys. Rev. E 48, 2553 (1993).

[29] R. M. Navarro and S. M. Fielding, Clustering and phase behaviour of attractive active particles with hydrodynamics, Soft Matter 11, 7525 (2015).

[30] T. Bickel, G. Zecua, and A. Würger, Polarization of active Janus particles, Phys. Rev. E 89, 050303 (2014).

[31] See Supplemental Material at http://link.aps.org/supplemental/ 10.1103/PhysRevE.94.012613 for typical trajectories of an artificial microswimmer.

[32] D. Takagi, A. B. Braunschweig, J. Zhang, and M. J. Shelley, Dispersion of Self-Propelled Rods Undergoing FluctuationDriven Flips, Phys. Rev. Lett. 110, 038301 (2013).

[33] P. Hänggi and H. Thomas, Stochastic processes: Time evolution, symmetries and linear response, Phys. Rep. 88, 207 (1982). See Sect. 2.4 therein for the inter-relationships among various stochastic calculus interpretations.

[34] H. Risken, The Fokker-Planck Equation (Springer, Berlin, 1984), Ch. 11.

[35] G. Costantini and F. Marchesoni, Threshold diffusion in a tilted washboard potential, Europhys. Lett. 48, 491 (1999).

[36] P. Reimann, C. Van den Broeck, H. Linke, P. Hänggi, J. M. Rubi, and A. Pérez-Madrid, Giant Acceleration of Free Diffusion by use of Tilted Periodic Potentials, Phys Rev. Lett. 87, 010602 (2001).

[37] P. Reimann, C. Van den Broeck, H. Linke, P. Hänggi, J. M. Rubi, and A. Perez Madrid, Diffusion in tilted periodic potentials: Enhancement, universality, and scaling, Phys. Rev. E 65, 031104 (2002).

[38] P. K. Ghosh, V. R. Misko, F. Marchesoni, and F. Nori, SelfPropelled Janus Particles in a Ratchet: Numerical Simulations, Phys. Rev. Lett. 110, 268301 (2013).

[39] P. E. Kloeden and E. Platen, Numerical Solution of Stochastic Differential Equations (Springer, Berlin, 1992)

[40] C. Festa, L. Fronzoni, P. Grigolini, and F. Marchesoni, The range of validity of the current procedures of adiabatic elimination: Experimental and theoretical evidence, Phys. Lett. A 102, 95 (1984).

[41] W. E. Schiesser, The Numerical Method of Lines: Integration of Partial Differential Equations (Academic Press, San Diego, 1991)

[42] E. Hairer and G. Wanner, Solving Ordinary Differential Equations II: Stiff and Differential-Algebraic Problems (Springer, Berlin, 1996)

[43] P. S. Burada, G. Schmid, and P. Hänggi, Entropic transport: A test bed for the Fick-Jacobs approximation, Philos. Trans. Roy. Soc. A Math. Phys. Eng. Sci. 367, 3157 (2009).

[44] P. Kalinay, Effective transport equations in quasi 1D systems, Eur. Phys. J.- Special Topics 223, 3027 (2014). 